\title{
Metaplasia e lesões histopatológicas de mucosa esofágica
}

\author{
Metaplasia and histopathological lesions of the esophageal mucosa \\ Metaplasia y lesiones histopatológicas de la mucosa esofágica
}

\author{
Elomar Rezende Moura \\ ORCID: https://orcid.org/0000-0002-1232-0146 \\ Universidade Tiradentes, Brasil \\ E-mail: elomar.rezende@ souunit.com.br \\ Gabrielle Barbosa Vasconcelos de Souza \\ ORCID: https://orcid.org/0000-0003-2465-356X \\ Universidade Tiradentes, Brasil \\ E-mail: gabriellebrbsa@gmail.com \\ Yasmin Tourinho Delmondes Trindade \\ ORCID: https://orcid.org/0000-0003-3054-9187 \\ Universidade Tiradentes, Brasil \\ E-mail: yasmindelmondes@gmail.com \\ Durval José de Santana Neto \\ ORCID: https://orcid.org/0000-0002-5730-5118 \\ Universidade Tiradentes, Brasil \\ E-mail: durvalneto777@gmail.com \\ Larissa Gonçalves Moreira \\ ORCID: https://orcid.org/0000-0002-5968-8634 \\ Universidade Tiradentes, Brasil \\ E-mail: larigoncalvesm@gmail.com \\ Wianne Santos Silva \\ ORCID: https://orcid.org/0000-0001-8006-8934 \\ Universidade Tiradentes, Brasil \\ E-mail: wiannessilva@gmail.com \\ Íkaro Daniel de Carvalho Barreto \\ ORCID: https://orcid.org/0000-0001-7253-806X \\ Universidade Federal de Pernambuco, Brasil \\ E-mail: daniel.carvalho.ib@gmail.com \\ Décio Fragata da Silva \\ ORCID: https://orcid.org/0000-0003-1157-6198 \\ Universidade Tiradentes, Brasil \\ E-mail: dfragata@yahoo.com.br \\ Luíse Meurer \\ ORCID: https://orcid.org/0000-0002-1315-4899 \\ Laboratório de Patologia Monica Araújo, Brasil \\ E-mail: luisemeurer@gmail.com \\ Leda Maria Delmondes Freitas Trindade \\ ORCID: https://orcid.org/0000-0003-4300-4274 \\ Universidade Tiradentes, Brasil \\ E-mail: ledeltrin@gmail.com
}

\section{Resumo}

A metaplasia é um processo adaptativo reversível, que acontece em um tipo de célula que, mediante um estímulo, passa pela substituição de outro tipo celular de linhagem semelhante. Objetivo: identificar a prevalência de metaplasia e a sua relação com lesões histopatológicas encontradas em amostras de mucosa esofágica. Método: estudo transversal, retrospectivo com uma amostra de 1953 laudos histopatológicos de mucosa esofágica. Resultados: de 1953 laudos, $548(28,1 \%)$ tinham metaplasia, sendo $94(17,1 \%)$ do tipo intestinal e destes 35(6,4\%) focal; 133(6,8\%) tinham esôfago de Barrett, 414 (75,5\%) com ectopia gástrica, $21(1,1 \%)$ com displasia, sendo $12(57,1 \%)$ de baixo grau e $9(42,9 \%)$ alto grau e $1427(73,1 \%)$ laudos apresentaram esofagite. A faixa etária mediana dos portadores de metaplasia foi de 45 anos (IIQ 34-55), sendo 289 (51,7\%) do sexo feminino e $438(81,4 \%)$ procedentes da capital. Dos laudos com metaplasia, 46 (8,2\%) tinham Helicobacter pylori, 30 (5,3\%) esôfago de Barrett e 14 (2,6\%) com atrofia gástrica associada a bactéria, entretanto não houve associação significativa com a H.pylori. Foi estatisticamente significativo $(\mathrm{p}<0,001)$ a associação de metaplasia com esofagite, esofagite eosinofílica e pólipo adenomatoso. Conjuntamente, observamos um menor risco de metaplasia na presença de esofagite (RR: 0,44; IC95\%: 0,36-0,55) e maior na presença de Eosinófilos >=15 eos/ CGA (RR: 3,27; IC95\%: 1,70-6,62). Conclusão: Não temos 
evidências de relação entre metaplasia no esófago e H.pylori. A ausência de esofagite e a presença de Eosinófilos >=15 eos/ CGA está associado a um maior risco de transformação metaplásica.

Palavras-chave: Mucosa esofágica; Metaplasia; Carcinogênese.

\begin{abstract}
Metaplasia is a reversible adaptive process, which occurs in a cell type that, upon a stimulus, undergoes the replacement of another cell type of similar lineage. Objective: to identify the prevalence of metaplasia in esophageal mucosal samples and evaluate the relationship between metaplasia and histopathological lesions. Method: crosssectional, retrospective study with a sample of 1953 histopathological reports of the esophageal mucosa. Results: from 1953 reports, $548(28.1 \%)$ had metaplasia, $94(17.1 \%)$ of the intestinal type and of these $35(6.4 \%)$ were focal; 133 (6.8\%) had Barrett's esophagus, 414 (75.5\%) with gastric ectopia, $21(1.1 \%)$ with dysplasia, $12(57.1 \%)$ with low grade and $9(42.9 \%)$ high degree and $1427(73.1 \%)$ reports presented esophagitis. The median age group of patients with metaplasia was 45 years (IIQ 34-55), with 289 (51.7\%) female and 438 (81.4\%) from the capital. Of the reports with metaplasia, $46(8.2 \%)$ had Helicobacter pylori, $30(5.3 \%)$ Barrett's esophagus and $14(2.6 \%)$ had gastric atrophy associated with bacteria, however there was no significant association with H. pylori. The association of metaplasia with esophagitis, eosinophilic esophagitis and adenomatous polyp was statistically significant $(\mathrm{p}<0.001)$. Together, we observed a lower risk of metaplasia in the presence of esophagitis (RR: 0.44 ; 95\% CI: $0.36-0.55$ ) and higher in the presence of Eosinophils $>=15$ eos/CGA (RR: 3.27; 95\%CI \%: 1.70-6.62). Conclusion: We have no evidence of a relationship between esophageal metaplasia and H.pylori. The absence of esophagitis and the presence of Eosinophils $>=15$ eos/CGA is associated with an increased risk of metaplastic transformation.
\end{abstract}

Keywords: Esophageal mucosa; Metaplasia; Carcinogenesis.

\title{
Resumen
}

La metaplasia es un proceso adaptativo reversible que ocurre en un tipo celular que, ante un estímulo, sufre el reemplazo de otro tipo celular de linaje similar. Objetivo: identificar la prevalencia de metaplasia en muestras de mucosa esofágica y evaluar la relación entre metaplasia y lesiones histopatológicas. Método: estudio transversal, retrospectivo, con una muestra de 1953 informes histopatológicos de la mucosa esofágica. Resultados: de 1953 informes, $548(28,1 \%)$ tenían metaplasia, $94(17,1 \%)$ de los cuales eran de tipo intestinal y de estos $35(6,4 \%)$ eran focales; 133 (6,8\%) tenían esófago de Barrett, 414 (75,5\%) con ectopia gástrica, 21 (1,1\%) con displasia, 12 (57,1\%) con grado bajo y $9(42,9 \%)$ grado alto y $1427(73,1 \%)$ informes presentados esofagitis. La mediana de edad de los pacientes con metaplasia fue de 45 años (IIQ 34-55), con $289(51,7 \%)$ mujeres y $438(81,4 \%)$ de la capital. De los informes con metaplasia, 46 (8,2\%) tenían Helicobacter pylori, $30(5,3 \%)$ esófago de Barrett y $14(2,6 \%)$ tenían atrofia gástrica asociada con bacterias, sin embargo, no hubo asociación significativa con $\mathrm{H}$. pylori. La asociación de metaplasia con esofagitis, esofagitis eosinofílica y pólipo adenomatoso fue estadísticamente significativa $(\mathrm{p}<0,001)$. Juntos, observamos un menor riesgo de metaplasia en presencia de esofagitis (RR: 0,44; IC del 95\%: 0,36-0,55) y mayor en presencia de eosinófilos> = 15 eos / CGA (RR: 3,27; IC del 95\%\%: 1,70-6,62). Conclusión: No tenemos evidencia de una relación entre la metaplasia esofágica y H. pylori. La ausencia de esofagitis y la presencia de eosinófilos $>=15$ eos / CGA se asocia con un mayor riesgo de transformación metaplásica.

Palabras clave: Mucosa esofágica; Metaplasia; Carcinogénesis.

\section{Introdução}

A metaplasia é um processo adaptativo reversível, que acontece em um tipo de célula (epitelial ou mesenquimal) sendo substituída por outro tipo celular da mesma linhagem. O crescimento celular de tecidos normais pode ser controlado ou não, localizado ou autolimitado e haver pequenas alterações nas células normais. Após cessado o estímulo o efeito é reversível a exemplo de lesões tipo hiperplasia, metaplasia e a displasia (Figura 1) (Inca, 2011).

Classifica-se a metaplasia segundo os achados histopatológicos e endoscópicos. A transformação do epitélio escamoso esofágico normal por um epitélio colunar especializado, como resposta ao refluxo gastroesofágico, seja por ácido gástrico e sais biliares, é fator de risco no processo da carcinogênese (Abbas, 2020). Esse processo, a partir de uma mutação genética (alteração no DNA celular) promove a proliferação de uma célula cancerosa. A exposição a vários agentes pode determinar o processo de oncogênese, entretanto as características individuais podem facilitar ou dificultá-lo (Inca, 2021). 
Figura 1. Processo de carcinogênese. Tipos de crescimento celular.

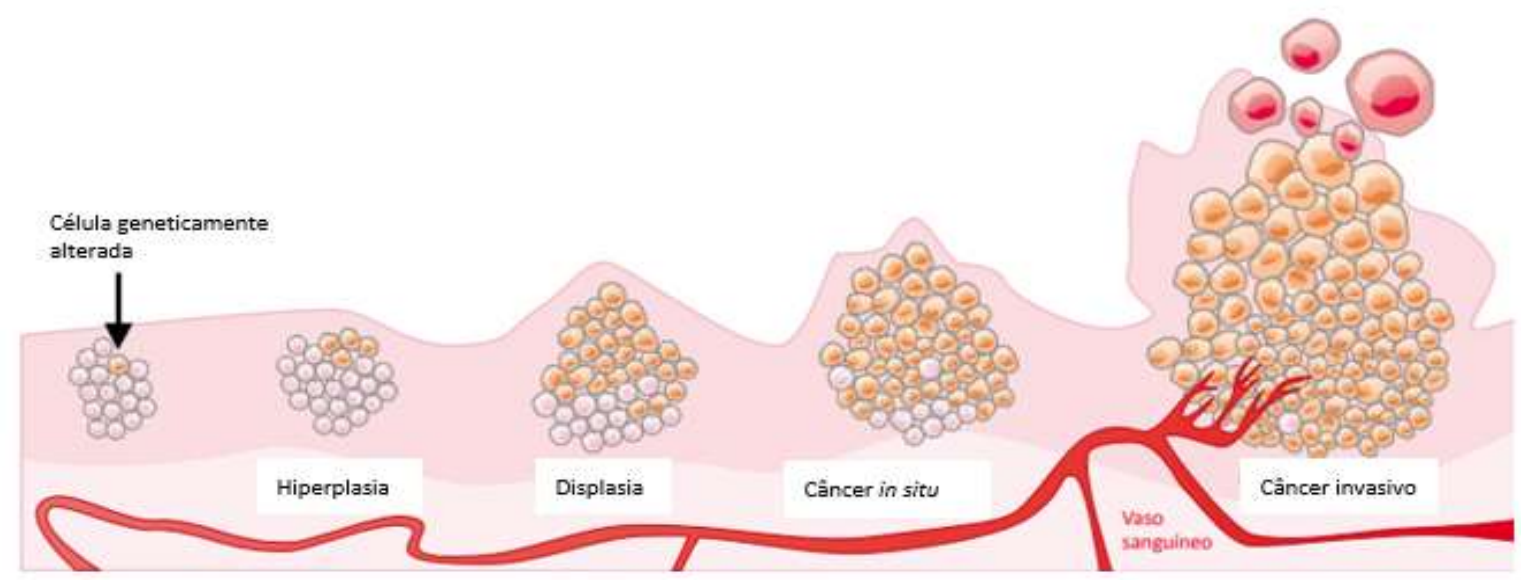

Fonte: Ilustração de Mariana F. Teles. Instituto Nacional de Câncer - Rio de Janeiro: Inca, 2011. 128. p.18.

A diferenciação celular provocada pela metaplasia é condicionada através da inativação de alguns genes. Sendo o esôfago um órgão que, em seu epitélio, não apresenta proteção contra a acidez gástrica, diversas complicações podem ocorrer. Uma das principais alterações encontradas é o esôfago de Barrett, que é caracterizado por ser uma condição patológica que aumenta consideravelmente a predisposição para o desenvolvimento de adenocarcinoma esofágico, um tipo de câncer com baixíssimo prognóstico (de Sá et al, 2020).

No Brasil, dentre os cânceres de esôfago, excetuando-se o câncer de pele não melanoma, o processo de carcinogênese esofágica ocupa a sexta posição como mais frequente entre homens e o décimo quinto lugar entre as mulheres. Sendo assim, o carcinoma epidermoide escamoso o tipo mais prevalente na população, sendo responsável pela grande maioria dos casos diagnosticados. Todavia, percebe-se um aumento em outro tipo de tumor: o adenocarcinoma. Estudos recentes de carcinogênese esofágica em ratos têm demonstrado que o câncer ocorre com maior frequência na presença de refluxo duodenal (Zhang, 2013). Cook (2011) concluiu que o efeito dos componentes do suco duodenal na carcinogênese esofágica, diante da ação isolada da bile, não aumenta a incidência de carcinoma, mas o suco pancreático isolado ou associado ao refluxo biliar o faz, mesmo na ausência de carcinógeno exógeno.

Segundo XIE, Fa-Jun et al (2013) em um estudo de meta-análise observou uma associação de infecção por helicobacter pylori e a alteração da mucosa esofágica. Os autores observaram que o risco de ESCC foi aumentado quando associado a infecção por H.pylori, enquanto que na população oriental houve uma diminuição estatisticamente significativa no risco de ESCC. Além disso, a microbiota pode estar associada ao esôfago de Barrett e adenocarcinoma esofágico ao interagir com seus fatores de risco, incluindo obesidade visceral, doença do refluxo gastroesofágico, presença da bactéria Helicobacter pylori, administração de inibidores da bomba de prótons e antibióticos (Lv et al (2019).

Diagnosticar lesões potencialmente malignas precocemente tem sido um árduo trabalho entre estudiosos quanto à etiologia infecciosa. Considerando as lesões comumente diagnosticadas em amostras de mucosa esofágica e a sua associação com metaplasia e a infecção por helicobacter pylori, este estudo teve como proposta identificar a prevalência de metaplasia em amostras de mucosa do esôfago, avaliar a relação entre metaplasia e as lesões histopatológicas e a correlação entre as lesões diagnosticadas e a bactéria helicobacter pylori.

\section{Metodologia}

Estudo transversal, retrospectivo, descritivo realizado de 2013 a 2018, na cidade de Aracaju, Sergipe, Brasil. A amostra foi composta por 1953 laudos histopatológicos de mucosa esofágica e mucosa gástrica, do banco de dados de uma 
clínica privada de anatomia patológica. O processo histológico de preparo das amostras ocorreu com inclusão em parafina e corados por Hematoxilina Eosina (HE) e, para a bactéria H.pylori, por Giemsa (HE). O instrumento de pesquisa foi composto pelas variáveis idade, sexo, procedência, atividade inflamatória, tipos de lesões em mucosa esofágica, presença ou não de Helicobacter pylori, metaplasia, displasias e neoplasias. Foram excluídos laudos com identificação incompleta e sem descrição das características histopatológicas das lesões identificadas. Utilizou-se o software R Core Team 2021 (versão 4.1.0) e o nível de significância adotado foi de 5\%. As variáveis categóricas foram descritas por frequência absoluta e relativa percentual e, as variáveis contínuas, por meio de mediana e intervalo quartil. A hipótese de independência entre variáveis categóricas testada por meio dos testes Qui-Quadrado de Pearson. A hipótese de aderência das variáveis contínuas à distribuição normal foi avaliada por meio do teste de Shapiro-Wilks. Como não foi confirmada, a hipótese de igualdade de duas medianas foi testada por meio do teste de Mann-Whitney. Foram estimados riscos relativos brutos e ajustados por meio da regressão de Poisson com erros padrões robustos simples e múltiplas. Para incluir variáveis no modelo ajustado foram utilizados os seguintes critérios: prevalência de ausentes inferior a 10\%, significância em teste de hipótese inferior ao nível de $20 \%$ e ausência de problemas de separação (caselas zeradas). Estudo aprovado pelo CEP/UNIT e parecer $n^{\circ} 1.246 .355$.

\section{Resultados}

Os laudos de biópsia de mucosa esofágica foram catalogados de acordo com os anos: 2013 (362), 2014 (371), 2015 (349), 2016 (237), 2017 (341), 2018 (293). Do total de 1953 laudos, 133(6,8\%) tinham esôfago de Barrett, 151 (7,7\%) eram positivos para HP, $1427(73,1 \%)$ apresentavam esofagite/processo inflamatório, 548 (28,1\%) tiveram diagnóstico de metaplasia, sendo $94(17,1 \%)$ do tipo intestinal e destes 35(6,4\%) focal (Figuras 2A e 2B). Metaplasia pancreática e ectopia gástrica em esôfago foram identificadas respectivamente em 87 (15,9\%) e 414 (75,5\%) das amostras.

Figura 2. Imagens histopatológicas de mucosa esofágica. Figura 1A. Junção de epitélio escamoso com epitélio glandular do tipo cárdico com infiltrado inflamatório mononuclear (HE,100X). Figura B. Área de esôfago de Barret. A seta indica glândula com células caliciformes características (HE 400X). Aracaju, 2013-2018.
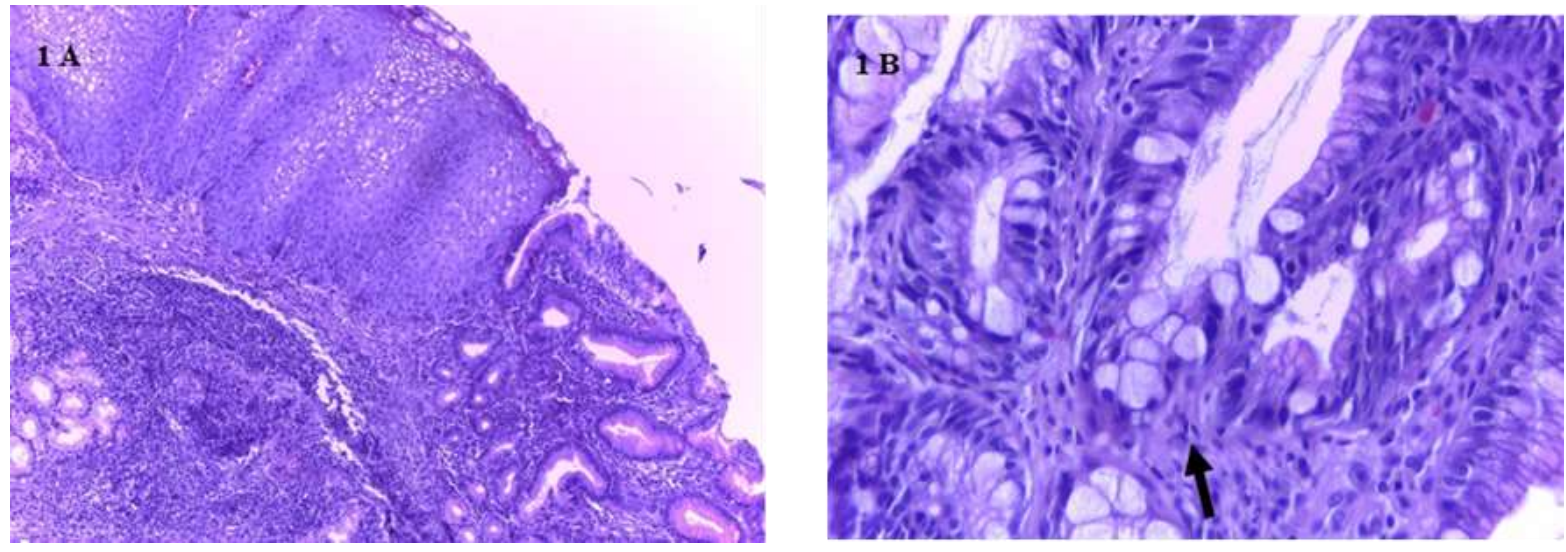

Fonte: Autores.

Lesões de displasia foram encontradas em $21(1,1 \%)$ laudos, sendo $12(57,1 \%)$ de baixo grau e $9(42,9 \%)$ alto grau. Outros achados histopatológicos: eosinófilos com $\mathrm{CGA}>/=15$ eos, hiperplasia epitelial, acantose glicogênica, xantelasma e pólipos

Dentre os portadores de metaplasia, a idade mediana foi de 45 anos (IIQ 34-55), sendo 289 (51,7\%) do sexo feminino e $438(81,4 \%)$ eram procedentes da capital. Do total daqueles que apresentaram metaplasia, $46(8,2 \%)$ tinham H. pylori positivo e $30(5,3 \%)$ esôfago de Barrett. Ao avaliarmos a hipótese de independência, correlacionando os aspectos 
epidemiológicos com metaplasia foram estatisticamente significativos ( $\mathrm{p}<0,05)$ a maior presença de esofagite em $483(85,6 \%)$ laudos, de pólipo adenomatoso em 2 (28,6\%) e menor de eosinófilos $>/=15$ eos/CGA em 8(1,4\%), hiperplasia epitelial em 10 $(1,8 \%)$ e pólipos em $7(1,2 \%)$ laudos. Dentre os laudos com metaplasia esofágica, 14 (2,6\%) tinham o diagnóstico de atrofia gástrica e não houve correlação significativa com a positividade para a bactéria H.pylori (Tabela 1).

Tabela 1. Aspectos epidemiológicos de portadores de metaplasia e sua associação com lesões de mucosa esofágica. Aracaju, 2013-2018.

\begin{tabular}{|c|c|c|c|}
\hline & \multicolumn{2}{|c|}{ Metaplasia } & \multirow[b]{2}{*}{ p-valor } \\
\hline & Sim & Não & \\
\hline Idade, Mediana (IIQ) & $45(33-55)$ & $44(33-56)$ & $0,154^{\mathrm{M}}$ \\
\hline \multicolumn{4}{|l|}{ Sexo, $n(\%)$} \\
\hline Feminino & $289(51,7)$ & $693(50,3)$ & $0,582^{Q}$ \\
\hline Masculino & $270(48,3)$ & $685(49,7)$ & \\
\hline \multicolumn{4}{|l|}{ Procedência, $n(\%)$} \\
\hline Capital & $438(81,4)$ & $1067(82,0)$ & $0,956^{\mathrm{Q}}$ \\
\hline Interior & $95(17,7)$ & $223(17,1)$ & \\
\hline Demais Estados & $5(0,9)$ & $12(0,9)$ & \\
\hline H. pylori & $46(8,2)$ & $105(7,6)$ & $0,655^{\mathrm{Q}}$ \\
\hline Esôfago de Barret & $30(5,3)$ & $103(7,4)$ & $0,096^{\mathrm{Q}}$ \\
\hline Heterotopia Gástrica, $n(\%)$ & $1(0,2)$ & $5(0,4)$ & $0,679^{Q}$ \\
\hline Esofagite, $n(\%)$ & $483(85,6)$ & $944(68,0)$ & $<0,001 \mathrm{Q}$ \\
\hline Eosinófilos >=15 eos/ CGA, $n(\%)$ & $8(1,4)$ & $70(5,0)$ & $<0,001 \mathrm{Q}$ \\
\hline Atrofia Gástrica & $14(2,6)$ & $27(2,1)$ & $0,516^{\mathrm{Q}}$ \\
\hline Hiperplasia Epitelial, $n(\%)$ & $10(1,8)$ & $51(3,7)$ & $0,031^{Q}$ \\
\hline Acantose Glicogênica, $n(\%)$ & $5(0,9)$ & $26(1,9)$ & $0,160^{Q}$ \\
\hline Xantelasma, $n(\%)$ & $0(0)$ & $3(0,2)$ & $0,269^{Q}$ \\
\hline Pólipos, $n(\%)$ & $7(1,2)$ & $40(2,9)$ & $0,032 \mathrm{Q}$ \\
\hline \multicolumn{4}{|l|}{ Localização/Região do Pólipo, $n$ (\%) } \\
\hline Proximal & $0(0)$ & $4(10,0)$ & $0,221^{Q}$ \\
\hline Médio & $0(0)$ & $1(2,5)$ & \\
\hline Distal & $4(57,1)$ & $7(17,5)$ & \\
\hline Não Descrita & $3(42,9)$ & $28(70,0)$ & \\
\hline \multicolumn{4}{|l|}{ Tipos de Pólipos, $n$ (\%) } \\
\hline Adenomatoso & $2(28,6)$ & $0(0)$ & $<0,001^{Q}$ \\
\hline \multicolumn{4}{|l|}{ Inflamatório não ulcerado(erosão) / } \\
\hline Hiperplásico não ulcerado (erosão) & $1(14,3)$ & $11(27,5)$ & \\
\hline \multicolumn{4}{|l|}{ Inflamatório ulcerado (erosão) / } \\
\hline Hiperplásico ulcerado(erosão) & $0(0)$ & $1(2,5)$ & \\
\hline Esofágico/Papiloma escamoso esofágico & $0(0)$ & $24(60,0)$ & \\
\hline Não descrito & $4(57,1)$ & $7(1,0)$ & \\
\hline
\end{tabular}

Legenda: $\mathrm{n}$ - frequência absoluta. \% - frequência relativa percentual. IIQ - intervalo Interquartil. M- Teste de Mann-Whitney. Q Teste Qui-Quadrado de Pearson. Fonte: Autores.

Foram ajustados riscos relativos brutos e ajustados para ocorrência de metaplasia. Identificou-se resultados significativos isoladamente para um menor risco de metaplasia na presença de esofagite (RR: 0,45; IC95\%: 0,37-0,56) e maior 
risco na presença de eosinófilos >=15 eos/ CGA (RR: 2,89; IC95\%: 1,49-5,59) e hiperplasia epitelial (RR: 1,79; IC95\%: 1,01$3,16)$. Além disso, foram identificados resultados significativos conjuntamente para um menor risco de metaplasia na presença de esofagite (RR: 0,44; IC95\%: 0,36-0,55) e maior na presença de eosinófilos >=15 eos/ CGA (RR: 3,27; IC95\%: 1,70-6,62) (Tabela 2).

Tabela 2. Lesões esofágicas que elevam o risco de desenvolver metaplasia. Aracaju, 2013-2018.

\begin{tabular}{lccc}
\hline & \multicolumn{2}{c}{ Metaplasia } & p-valor \\
\cline { 2 - 3 } Esôfago de Barrett & RR (IC95\%) & RRa (IC95\%) & \\
Esofagite & $1,30(0,94-1,80)$ & & $<0,001$ \\
Eosinófilos $>=15$ eos/ CGA & $0,45(0,37-0,56)$ & $0,44(0,36-0,55)$ & $<0,001$ \\
Hiperplasia Epitelial & $2,89(1,49-5,59)$ & $3,27(1,70-6,62)$ & \\
Pólipos & $1,79(1,01-3,16)$ & & \\
\hline
\end{tabular}

Legenda: RR - Risco Relativo. RRa - Risco Relativo Ajustado. IC95\% - Intervalo com 95\% de confiança. Fonte: Autores.

\section{Discussão}

A prevalência de metaplasia encontrada em laudos histopatológicos de esôfago foi em torno $28,1 \%$ (548) e o sexo feminino foi o mais prevalente. Considerando o total da amostra, 6,8\% tinham esôfago de Barrett, 7,7\% eram positivos para helicobacter pylori, $73,1 \%$ apresentaram processo inflamatório/esofagite e $28,1 \%$ tinham metaplasia. Dentre os tipos de metaplasia identificados, a metaplasia intestinal foi percentualmente mais frequente, sendo $6,4 \%$ do tipo focal. Achados como metaplasia pancreática $(15,9 \%)$ e ectopia gástrica $(75,5 \%)$ também foram registrados.

Sabe-se que a metaplasia intestinal apresenta características peculiares. A presença constante de ácido clorídrico em terço inferior do esôfago representa estímulo para a substituição do epitélio escamoso estratificado pelas células caliciformes, promovendo estado de metaplasia. Tem sido atribuído um efeito protetor à bactéria H.pylori nos portadores de DRGE, esofagites, esôfago de Barrett e adenocarcinoma esofágico, embora ainda seja controverso (Kusters et., al 2006)

Diversos fatores podem contribuir para o estado de cronicidade da DRGE e consequentemente desenvolvimento de metaplasia intestinal em esôfago distal. Embora a literatura aponte como incerta, a bactéria $\mathrm{H}$. pylori tem sido associada a patologias que cursam com refluxo ácido esofágico, ao observar que, em função da redução da secreção ácida do estômago, devido a colonização da bactéria, esta estaria favorecendo a redução da produção ácida pelo estômago e consequentemente redução do refluxo ácido gastroesofágico. Autores (Henrik Simán et al., 2001) citam que a associação entre a infecção por Helicobacter pylori e neoplasias esofágicas parece haver um risco reduzido em desenvolver a doença.

A faixa etária mediana dos portadores de metaplasia foi de 45 anos (IIQ 34-55), sendo 51,7\% (289) do sexo feminino. Os autores avaliaram o efeito da bactéria H.pylori em relação a metaplasia intestinal quanto ao gênero e idade e observaram que não houve associação significativa entre a bactéria e metaplasia intestinal.

Do total daqueles que apresentavam metaplasia, 8,2\%(46) tinham $\mathrm{H}$. pylori positivo e 5,3\% (30) esôfago de Barrett. Os dados do estudo demonstraram que a bactéria H.pylori em associação com metaplasia não demonstrou fator de risco para metaplasia esofágica. Degiovani, et al. (2019) também demonstraram que a probabilidade de ocorrer metaplasia intestinal em mucosa esofágica, em portadores ou não de H. pylori, não houve significância estatística.

Acreditava-se que a bactéria Helicobacter pylori exercia, na mucosa gástrica, um papel importante na formação carcinogênica, enquanto que, a sua ação direta na mucosa esofágica não era evidente. Entretanto, evidências têm indicado que a infecção pela bactéria exerce papel na patogênese de doenças do aparelho digestivo e dentre elas encontra-se o adenocarcinoma de esôfago (Andreollo et al, 2018). 
A ausência de consenso nas publicações a respeito do tema, tem dificultado definir a ação direta ou indireta da bactéria nas patologias esofágicas. Infere-se que as diversas metodologias aplicadas possam deixar brechas para tal incerteza. Observou-se uma redução do risco de carcinoma de células escamosas de esôfago (CCEE) e adenocarcinoma de esôfago (EAC) ao relacionar a bactéria Helicobacter pylori e o risco de carcinoma de esôfago (XIE, Fa-Jun et al., 2013). Estudo populacional de meta análise analisou isoladamente e estratificados por local de estudo, a associação de câncer do esôfago e a infecção por H.pylori, em grupos populacionais ocidentais e orientais. Observou-se uma heterogeneidade quanto ao risco relativo entre esses grupos, atribuindo a cepas e ao ambiente (Degiovani et al, 2019).

Ao demonstrar a correlacionar de metaplasia com outras lesões histopatológicas da mucosa esofágica, observou-se que algumas alterações histopatológicas apresentaram uma associação significativa, enquanto fator de risco e chance de metaplasia, em lesões como Esôfago de Barrett, esofagite eosinofílica, hiperplasia epitelial e pólipos. Mais de 73\% dos laudos apresentaram esofagite e, nesse estudo, a esofagite não parece ser fator de risco isolado quando associado com a metaplasia. Entretanto, autores apontam que a esofagite crônica parece ser a lesão inicial da história natural do carcinoma epidermóide de esôfago, passando pelos sucessivos estados de esofagite crônica, atrofia, displasia e câncer (Daly et al, 2000). Estudo desenvolvido por Fonseca et al (2021) observou que a metaplasia isoladamente não apresentou significância estatística, no entanto, quando associado a outros achados, a inflamação crônica estava presente em todos os portadores com metaplasia.

A displasia encontrada em laudos com diagnóstico de metaplasia da mucosa esofágica foi representada por $57,1 \%$ de baixo grau e 42,9\% de alto grau. Quando a displasia é observada em laudos histopatológicos, atribui-se a estreita relação com carcinoma epidermóide do esôfago, não existindo diferenças substanciais entre sua atividade celular proliferativa e a do carcinoma intraepitelial (Terry et al, 2000; Siewert et al (2001). No adenocarcinoma, a evolução se dá através de uma série de alterações progressivamente displásicas no epitélio colunar (Gammon, 1997), e a progressão ocorre a partir da metaplasia especializada de Barrett, para anormalidades no indefinido espectro das displasias de baixo grau, alto grau e, por fim, carcinoma (Vaughan et al, 1995).

Outros achados histopatológicos foram identificados em associados com a presença de metaplasia, tais como: esofagite eosinofílica, atrofia gástrica, hiperplasia epitelial, acantose glicogênica, xantelasma e pólipos. Nas amostras que apresentaram positividade para $\mathrm{H}$. pylori, buscou-se avaliar os laudos de mucosa gástrica do mesmo paciente, no sentido de observar a presença de atrofia gástrica. Entretanto, não se observou associação significativa com a presença da bactéria helicobacter pylori.

Ao avaliar a correlação de fatores de risco e a chance de metaplasia, observou-se que a probabilidade de uma lesão em mucosa esofágica desenvolver metaplasia indicou uma associação positiva ou risco aumentado em relação aos não expostos tais como esôfago de Barrett, esofagite eosinofílica, hiperplasia epitelial e pólipos.

Não foram encontrados estudos relacionando esofagite eosinofílica em associação com e metaplasia. Neste estudo, identificou-se resultados significativos para um maior risco de metaplasia na presença de eosinófilos (RR: 2,89; IC95\%: 1,495,59) e hiperplasia epitelial (RR: 1,79; IC95\%: 1,01-3,16). Em vários processos inflamatórios observa-se hiperplasia de células da camada basal. Estudos citam que a esofagite eosinofílica pode se apresentar em associação com DRGE (Steiner et al., 2006). Ora, se ocorre um processo inflamatório constante e morfologicamente ocorre proliferação de células da zona basal, a renovação constante do epitélio pode comprometer a lâmina própria podendo levar progressivamente a uma remodelação esofágica. Poderíamos assim pensar que seria uma contribuição para desenvolver metaplasia (Aceves, 2014).

Embora a presença de esofagite tenha sido estatisticamente significativa dentre as lesões encontradas em mucosa esofágica, ao correlacionar com metaplasia observou-se que há uma associação inversa ou um risco diminuído exposto ao fator estudado.

A transformação maligna envolve diversas etapas e é regulada por muitos genes implicados no processo 
carcinogênico (Souza, 2002; Dolan et al 2003). Diferentes lesões genômicas têm sido reconhecidas na displasia do epitélio esofágico, frente a oncogênese e genes supressores tumorais (Casson et al, 2003).

A literatura apresenta poucos estudos em relação a achados histopatológicos da mucosa esofágica e metaplasia. Os estudos buscam a correção de metaplasia com DRGE e com esôfago de Barrett e a bactéria H pylori e carcinogênese. É possível que estudos populacionais possam colaborar substancialmente para avaliar com maior acurácia a associação de metaplasia e lesões esofágicas e que fatores etiológicos estariam diretamente associados no processo de carcinogênese do esôfago.

Como limitação, se tratando de um estudo observacional, são esperados vieses de seleção de pacientes. Uma vez que a busca pela biópsia geralmente parte de queixas apresentadas pelo paciente, é possível inferir que as prevalências de achados estejam maiores do que na população geral o que não permite uma generalização para toda população, mas não impede para população com queixa.

\section{Conclusão}

A metaplasia, enquanto processo adaptativo das células, foi observado principalmente em associação com esofagite, esofagite eosinofílica e pólipo adenomatoso. Não se observou prevalência entre os sexos. Ao correlacionar os achados histopatológicos com metaplasia, observou-se que lesões histopatológicas como esôfago de Barrett, esofagite eosinofílica, hiperplasia epitelial e pólipo adenomatoso demonstraram alguma probabilidade de risco em relação a transformação metaplásica. Conjuntamente, a ausência de esofagite e a presença de eosinófilos >=15 eos/ CGA está associado a um maior risco de transformação metaplásica. Sendo assim, outras pesquisas devem ser conduzidas para avaliar a interação de diversos fatores, tais como características individuais, tempo de exposição a agentes carcinogênicos e frequência.

\section{Referências}

Abbas, A. K. et al. (2008). Robbins patologia básica. Elsevier Brasil.

Aceves S. S. (2014). Remodeling and fibrosis in chronic eosinophil inflammation. Digestive diseases (Basel, Switzerland), 32(1-2), 15-21. https://doi.org/10.1159/000357004.

Andreollo, N. A., Beraldo, G. D. C., Alves, I. P. F., Tercioti-Junior, V., Ferrer, J. A. P., Coelho-Neto, J. D. S., \& Lopes, L. R. (2018). Pathologic Complete Response (YPT0 YPN0) after chemotherapy and radiotherapy neoadjuvant followed by esophagectomy in the squamous cell carcinoma of the esophagus. ABCD. Arquivos Brasileiros de Cirurgia Digestiva (São Paulo), 31.

Casson, A. G., Evans, S. C., Gillis, A., Porter, G. A., Veugelers, P., Darnton, S. J., ... \& Hainaut, P. (2003). Clinical implications of p53 tumor suppressor gene mutation and protein expression in esophageal adenocarcinomas: results of a ten-year prospective study. The Journal of thoracic and cardiovascular surgery, 125(5), 1121-1131.

Cook, M. B. (2011). Non-acid reflux: the missing link between gastric atrophy and esophageal squamous cell carcinoma? Am J Gastroenterol. 106: 1930-2. [PMID: 22056574 DOI: 10.1038/ajg.2011.288].

Daly, J. M., Fry, W. A., Little, A. G. et al. (2000). Esophageal cancer: results of an American College of Surgeons Patient Care Evaluation Study. J Am Coll Surg. 190: 562-72.

de Sá, R. C., de Sá, L. K. D. S., Moreno, V. G., de Medeiros Araújo, D. C., \& Teston, A. P. M. (2020). Incidência comprobatória de esôfago de barrett (metaplasia) com hipótese de diagnóstico inicial em um laboratório patológico do norte do Paraná. Brazilian Journal of Development, 6(10), $77720-77727$.

Degiovani, M et. al, (2019). Existe relação entre o Helicobacter pylori e metaplasia intestinal nas epitelizações colunares curtas até 10 mm no esôfago. 32(4): e1480. DOI: /10.1590/0102-672020190001e1480 distal? https://www.scielo.br/j/abcd/a/Jzwq56NC68yPnmmxqynQRpD/?format=pdf\&lang=pt11.

Dolan, K., Walker, S. J., Gosney, J., Field, J. K., \& Sutton, R. (2003). TP53 mutations in malignant and premalignant Barrett's esophagus. Diseases of the esophagus : official journal of the International Society for Diseases of the Esophagus, 16(2), 83-89. https://doi.org/10.1046/j.1442-2050.2003.00302.x.

Gammon, M. D., Schoenberg, J. B., Ahsan, H., Risch, H. A., Vaughan, T. L., Chow, W. H., Rotterdam, H., West, A. B., Dubrow, R., Stanford, J. L., Mayne, S. T., Farrow, D. C., Niwa, S., Blot, W. J., \& Fraumeni, J. F., Jr (1997). Tobacco, alcohol, and socioeconomic status and adenocarcinomas of the esophagus and gastric cardia. Journal of the National Cancer Institute, 89(17), 1277-1284. https://doi.org/10.1093/jnci/89.17.1277.

Henrik Simán, J., Forsgren, A., Berglund, G., Florén, C. H. (2001) Helicobacter pylori infection is associated with a decreased risk of developing oesophageal neoplasms. Helicobacter;6(4):310-6. Available from: http://www.ncbi.nlm.nih.gov/pubmed/11843963. 
Research, Society and Development, v. 11, n. 2, e29711225778, 2022

(CC BY 4.0) | ISSN 2525-3409 | DOI: http://dx.doi.org/10.33448/rsd-v11i2.25778

INCA. (2021). Como surge o câncer? Instituto Nacional do Câncer. https://www.inca.gov.br/como-surge-o-cancer.

Instituto Nacional de Câncer (2011). ABC do câncer: abordagens básicas para o controle do câncer / Instituto Nacional de Câncer. Rio de Janeiro. Inca. 128 p. : il

Kusters, J. G., Van Vliet, A. H. M. \& Kuipers, E. J. (2006) Patogênese da infecção por Helicobacter pylori. Clinical Microbiology Reviews, 19, 449-90. http://dx.doi.org/10.1128/CMR.00054-05 http://www.ncbi.nlm.nih.gov/pubmed/16847081.

Lv, J., Guo, L., Liu, J. J., Zhao, H. P., Zhang, J., \& Wang, J. H. (2019). Alteration of the esophageal microbiota in Barrett's esophagus and esophageal adenocarcinoma. World journal of gastroenterology, 25(18), 2149.

Sales, K. M. S., Silva, W. S., Carvalho, G. P. S. de, Fonseca, A. M. L., Silva, G. P. O., Viaggi, T. C., Aragão, B. C., Fonseca, A. M. R., Braga, A. F. L. da R., Chagas, A. M. B., Guimarães, B. M. de A., Trindade, L. M. D. F., \& Lima, F. S. de. (2021). Achados endoscópicos em portadores de sintomas dispépticos. Research, Society and Development, 10(13), e562101321619. https://doi.org/10.33448/rsd-v10i13.21619.

Siewert, J. R., Stein, H. J., Feith, M., Bruecher, B. L., Bartels, H., \& Fink, U. (2001). Histologic tumor type is an independent prognostic parameter in esophageal cancer: lessons from more than 1,000 consecutive resections at a single center in the Western world. Annals of surgery, 234(3), 360-369. https://doi.org/10.1097/00000658-200109000-00010.

Souza R. F. (2002). Molecular and biologic basis of upper gastrointestinal malignancy--esophageal carcinoma. Surgical oncology clinics of North America, 11(2), 257-viii. https://doi.org/10.1016/s1055-3207(02)00003-0.

Steiner, S. J., Kernek, K. M., \& Fitzgerald, J. F. (2006). Severity of basal cell hyperplasia differs in reflux versus eosinophilic esophagitis. Journal of pediatric gastroenterology and nutrition, 42(5), 506-509. https://doi.org/10.1097/01.mpg.0000221906.06899.1b.

Terry, P., Lagergren, J., Ye, W., Nyrén, O., \& Wolk, A. (2000). Antioxidants and cancers of the esophagus and gastric cardia. International journal of cancer, $87(5), 750-754$

Vaughan, T. L., Davis, S., Kristal, A., \& Thomas, D. B. (1995). Obesity, alcohol, and tobacco as risk factors for cancers of the esophagus and gastric cardia: adenocarcinoma versus squamous cell carcinoma. Cancer epidemiology, biomarkers \& prevention : a publication of the American Association for Cancer Research, cosponsored by the American Society of Preventive Oncology, 4(2), 85-92.

Xie, F-J. et al. (2013). Helicobacter pylori infection and esophageal cancer risk: an updated meta-analysis. World journal of gastroenterology: WJG. 19(36), 6098.

Zhang, Y. (2013). Epidemiology of esophageal cancer. World journal of gastroenterology: WJG. 19(34), 5598. 